\title{
Acoustic analysis of voice using WPCVox: a comparative study with Multi Dimensional Voice Program
}

\author{
Juan Ignacio Godino-Llorente • Víctor Osma-Ruiz • \\ Nicolás Sáenz-Lechón - Ignacio Colreta-Marco · \\ Ramón González-Herranz · Carlos Ramírez-Calvo
}

\begin{abstract}
Alsstract In this study, two different tools developed for the parametric extraction and acoustic analysis of voice samples are compared. The main goal of the paper is to contrast the results obtained using the classical Multi Dimensional Voice Program (MDVP), with the results obtained with the novel WPCVox. The aim of this comparison was to find differences and similarities in the parameters extracted with both systems in order to make comparison of measurements and data transfer among both equipments. The study was carried out in two stages: in the first, a wide sample of healthy voices belonging to Spanishspeaking adults from both genders were used to carry out a direct comparison between the results given by MDVP and those obtained with WPCVox. In the second stage, a sample of 200 speakers ( 53 normal and 173 pathological) taken from a commercially available database of voice disorders were used to demonstrate the usefulness of WPCVox for the acoustic analysis and the characterization of normal and pathological voices. The results conclude that WPCVox provides very reliable measurements which are very similar to those obtained using MDVP, and very similar capabilities to discriminate among normal and pathological voices.
\end{abstract}

Keywords Normal voice Pathological voice Acoustic measurements - WPCVox · MDVP

\section{Introduction}

The present work deals with one of the most complete instruments available for the acoustic analysis of voice: the Multi Dimensional Voice Program (MDVP), developed by Kay Elemetrics , in comparison with a novel commercial tool that has recently been made available in the market: WPCVox . Both systems are capable of rapidly calculating a large number of parameters to measure different aspects of the vocal function, facilitating the graphical representation of these parameters. Despite of the similarities among both systems, small differences in the estimation of the parameters are expected as those existing between MDVP and the other de facto standard in the field of the acoustic analysis: Dr. Speech

Throughout this paper the normative values are given for the main acoustic parameters given by MDVP and WPCVox with Spanish-speakers. At the same time, the parameters calculated by both systems are compared in order to determine whether the parameters calculated are measuring the same phenomenon or not. Moreover, for the sake of comparison, the discrimination capabilities to separate normal and pathological voices have been analysed with both sets of equipment.

\section{Material and methods}

Selection of the corpus speakers

In order to represent the normality criteria, 520 voice samples corresponding to non pathological speakers were 
recorded at the "Principe de Asturias" Hospital in Alcalá de Henares, Madrid. From this dataset 356 speakers were selected matching the inclusion requirements fixed for the study. The distribution by gender is: 226 females and 130 males. The age range goes from 9 to 79 years old, with a mean of 35.5 years. For each speaker, the acoustically balanced utterance "es hábil un solo día" was recorded, together with the sustained phonation of vowel ' $a$ ' for a duration of at least $3 \mathrm{~s}$.

The inclusion criteria fixed to select the speakers to be part of the final corpus were: (a) The subjective feeling that the speaker has no perceived laryngeal pathology; (b) An adequate voice for the age, gender and cultural group of the speaker, and an adequate pitch, timbre, volume and flexibility of diction; (c) Non smoker; (d) The speaker has never undergone any surgical operation related with any laryngeal pathology; (e) The speaker has not required endotracheal intubations in the last year; (f) A perceptual judgement according to the GRBAS scale with a score below 3 .

The corpus was complemented with pathological voices from a wide range of disorders. The same material was recorded for those patients that visited the ENT service of the mentioned Hospital. The pathological dataset contains 203 samples with a wide range of pathologies. Such pathological dataset was not used in this study but will be helpful for future research.

For the second part of the study presented in this paper, we have used the Voice Disorders Database distributed by Kay Elemetrics. Two hundred and twenty six samples were selected according to the criteria explained

(53 normal and 173 pathological). This database was used to test the usefulness of WPCVox for acoustic analysis and discrimination among normal and pathological voices. The experiment was carried out with the database distributed by Kay Elemectrics because it is easily available and it would allow the reproducibility of the results.

The explanation to the fact that the databases used have been recorded with the MDVP is that in order to compare the algorithms implemented in both equipments, the speech files had to be recorded with the same system to avoid differences that could appear due to the A/D converter or sampling rates.

\section{Description of the exploration techniques}

The exploration techniques followed to obtain the normal voices consist of several steps, first of all, the vocal anamnesis is carried out, paying special attention to those factors that do not match the inclusion criteria enumerated before; after the anamnesis, a stroboscopy with a telelaryngoscope was usually carried out to dismiss any laryngeal pathology, then the microphonic joint to the electroglottographic signal was recorded. A condenser microphone was used, placed on a stand at $30 \mathrm{~cm}$ from the mouth at a $50^{\circ}$ angle. The recordings were performed with the CSL $4300 \mathrm{~B}$ equipment of Kay Elemetrics, sampling both signals with a frequency of $50 \mathrm{kHz}$ and 16 bits of quantization. All the recordings were made under the same conditions, using macros to ensure an identical configuration of the configuration parameters. All the samples were collected in a soundproof room. The samples correspond to the sustained phonation of the Spanish vowel ' $a$ '. The first $500 \mathrm{~ms}$. and the last part of the utterance were removed to avoid onset and offset effects. Finally, only $3 \mathrm{~s}$. (the midvowel segment) was used for further analysis.

Regarding the second stage of the work, 173 pathological and 53 normal speakers were selected according to the criteria fixed. After edition and selection of the stationary part in the middle of the sustained phonation, the files were stored in the database by the developers with a sampling frequency of $50 \mathrm{kHz}$. The duration of the pathological records is around $0.8 \mathrm{~s}$. long, whereas the normal voices are $3 \mathrm{~s}$. long. The samples correspond to the sustained phonation of the vowel 'ah'.

\section{Instrumentation: MDVP and WPCVox equipments}

The MDVP is a part of the CSL-4300B equipment. It is probably one of the most standardized systems currently existing in the market. The equipment has its own input/ output recording device connected to a $\mathrm{PC}$ by means of a PCI hardware interface. The hardware has been developed to simultaneously record four signals with a maximum sampling frequency of $51.2 \mathrm{kHz}$. With its integrated hardware and sottware, it is tailored for sound input and measurement in speech processing applications. The system records different types of signals (electroglottographic, microphonic, and inverse filtered signals) providing the possibility of using several sottware modules that complement the analysis. The MDVP provides up to 24 parameters (frequency and amplitude perturbation, noise, tremor parameters, etc) providing a multidimensional analysis of the voice. The most important parameters calculated by MDVP are summarized in Table 1.

WPCVox is an application developed with the aim of recording and analyzing speech and electroglottographic (EGG) signals. This is a low-cost commercial application that just becomes available to the scientific community. WPCVox is one of the first commercial applications developed

\footnotetext{
${ }^{1}$ Not all the patients were subjected to an endoscopy: only those presenting a vocal disorder based on a previous psychoacoustic judgement of theit voice. In total. 200 patients underwent these explotation techniques.
} 
Table 1 Description of the acoustic measurements performed by WPCVox and MDVP

\begin{tabular}{|c|c|c|c|c|}
\hline Туре & Parameter & Description & MDVP & WPCVox \\
\hline \multirow{6}{*}{$\begin{array}{l}\text { Frequency } \\
\text { perturbations }\end{array}$} & fo & Average fundamental frequency for the vocalization $(\mathrm{Hz})$. & $\times$ & $\times$ \\
\hline & Jitt abs & $\begin{array}{l}\text { Absolute jitter gives an evaluation of the period-to-period } \\
\text { variability of the pitch period (ms). }\end{array}$ & $\because$ & $\times$ \\
\hline & Jitt rel & $\begin{array}{l}\text { Jitter percent represents the relative period-to-period } \\
\text { (in short-term) variability of the pitch (\%). }\end{array}$ & $\because$ & $\times$ \\
\hline & RAP & $\begin{array}{l}\text { Relative average perturbation gives the variability of the } \\
\text { pitch period with a smoothing factor of three periods }(\%) \text {. }\end{array}$ & $\times$ & $\therefore$ \\
\hline & $\mathrm{PPQ}$ & $\begin{array}{l}\text { Pitch period perturbation quotient gives the variability of the } \\
\text { pitch period with a smoothing factor of } 5 \text { periods (\%). }\end{array}$ & $\because$ & $\times$ \\
\hline & $\mathrm{sPPQ}$ & $\begin{array}{l}\text { Smoothed PPQ gives the long-term variability of the pitch } \\
\text { period with a user-selected number of periods (usually } 55)(\%) \text {. }\end{array}$ & $\times$ & $\therefore$ \\
\hline \multirow[t]{4}{*}{$\begin{array}{l}\text { Amplitude } \\
\text { perturbations }\end{array}$} & Shimm abs & $\begin{array}{l}\text { Shimmet in } \mathrm{dB} \text { gives the period-to-period vatiability of the } \\
\text { peak-to-peak amplitude within the analysed voice sample (dB). }\end{array}$ & $\times$ & $\therefore$ \\
\hline & Shimm rel & $\begin{array}{l}\text { Shimmer percent gives the variability of the peak-to-peak } \\
\text { amplitude. It represents the relative period-to-period } \\
\text { (in short-term) variability of the peak-to-peak amplitude }(\%) \text {. }\end{array}$ & $\because$ & $\times$ \\
\hline & $\mathrm{APQ}$ & $\begin{array}{l}\text { Amplitude perturbation quotient gives the variability of the } \\
\text { peak-to-peak amplitude with a smoothing factor of } 11 \text { periods (\%). }\end{array}$ & $\times$ & $\therefore$ \\
\hline & sAPQ & $\begin{array}{l}\text { Smoothed APQ gives the long-term variability of the peak-to-peak } \\
\text { amplitude with a user-selected number of periods (usually } 55 \text { ) (\%). }\end{array}$ & $\because$ & $\times$ \\
\hline \multirow[t]{4}{*}{ Noise } & NHR & $\begin{array}{l}\text { Noise-to-harmonics ratio is an average ratio of enetgy of the } \\
\text { in-harmonic components in the range } 1,500-4.500 \mathrm{~Hz} \text { to the } \\
\text { harmonic components energy in the range } 70-4.500 \mathrm{~Hz} \text { (\%). }\end{array}$ & $\times$ & $\therefore$ \\
\hline & HNR & $\begin{array}{l}\text { Harmonics-to-noise ratio is an average ratio of the energy of the } \\
\text { harmonics related to the noise energy in the range } 70-4.500 \mathrm{~Hz}(\mathrm{~dB}) \text {. }\end{array}$ & & $\times$ \\
\hline & NNE & $\begin{array}{l}\text { Normalized noise energy is a ratio of the energy of the noise } \\
\text { present above } 1 \mathrm{kHz} \text { to the total energy of the signal (dB). }\end{array}$ & & $\times$ \\
\hline & VTI & $\begin{array}{l}\text { Voice turbulence index is an average ratio of the spectral in-harmonic } \\
\text { high-frequency energy in the range } 2.800-5,800 \mathrm{~Hz} \text { to the spectral } \\
\text { harmonic energy in the range } 70-4,500 \mathrm{~Hz}(\%) \text {. }\end{array}$ & $\because$ & $\because$ \\
\hline
\end{tabular}

in Europe for the objective analysis of voice disorders. The recording is carried out using an external standard sound card (SoundBlaster Live 24 bit External) connected to a USB port that gives a signal to noise ratio (SNR) over $100 \mathrm{~dB}$ with 24 bits of resolution and sampling frequencies above $48 \mathrm{kHz}$. This software tool permits the synchronous recording of speech and EGG signals using an active connector specially developed to mix both signals together. The application is able to extract and represent graphically the most useful parameters for evaluating voice quality (Table 1).

\section{Statistics}

For each parameter, the samples used to compare the results obtained with both sets of equipment were selected within three times the interquartile range, the samples falling outside this range being regarded as outlayers due to measurement errors. For testing the discrimination capabilities no samples were rejected.
For the sake of comparison, parametric (Pearson correlation coefficient ) and nonparametric tests (Kendall and Spearman correlation coefficients ) were carried out, considering that there exists a statistical significance with a $P$ value below 0.05 (95\% confidence ${ }^{2}$ ). Moreover, the significance between the differences among the two programs was measured using the $t$-test considering that there exists statistical significance with a $P$ value below 0.05 ( $95 \%$ confidence). In the parametric tests, when it was necessary due to the shape of the underlying distribution, the parameters were transformed to match the criteria of gaussianity and homocedasticity using a neperian logarithmic transformation: $\ln (\cdot)$. Normality was tested using the Kolmogorov-Smirnov test considering that there exists statistical significance with a $P$ value above $0.05(95 \%$ confidence)-, and $\mathrm{Q}-\mathrm{Q}$ plots . This transformation allows

\footnotetext{
${ }^{2}$ Contrasting is more confident evaluating the statistical significance with $95 \%$ than $99 \%$.
} 
the use of parametric tests such as Student's $t$. This solution has been used previously by other authors

The comparison using correlations among measurements has been used by other authors before . A high correlation is a necessary condition to say that both algorithms are similar. The sufficient condition is found in the fact that the measurements are based on the same grounds and algorithms.

All statistical analyses were performed in SPSS $12.0 \mathrm{~S}$ for Windows (SPSS Corporation, Chicago, IL, USA, 2003).

\section{Results}

The comparative results are grouped into two sections. The first deals with the similarities between the values of the parameters obtained with both systems and the calculation of the normative values. The second is a comparison of the discrimination capabilities of both sets of equipment.

\section{Normative values}

This section compares the values obtained for the fundamental frequency, the amplitude and frequency perturbations, and the noise parameters; all of them calculated using both systems. The comparison is done by means of the correlation coefficients among those parameters with a similar definition. Table 5 summarises the normative values for MDVP and WPCVox, giving the mean, mode, the 75 and $90 \%$ percentiles. The mode values were included because the histograms do not follow a Gaussian distribution.

\section{Fundamental frequency (fo)}

The mean fundamental frequency (fo) obtained with WPCVOx is around $120 \mathrm{~Hz}$ for males and $200 \mathrm{~Hz}$ for females, both groups following a Gaussian distribution. As expected, significant variations have been found in both groups $(P<0.001)$. Table 2 shows the mean $(\mu)$, standard deviation $(\sigma), 1$ and $99 \%$ percentiles of the fo separated by gender for both equipments. In terms of comparison, the results obtained using both equipments are almost identical, presenting a high correlation coefficient $(r=0.98$, according to Pearson).

\section{Frequency perturbation: jitter parameters}

The measurement with MDVP gives a mean value of jitt abs of $58.85 \mathrm{~ms}$, with a wide range of variation. The jitt abs measured with WPCVox is $67.77 \mathrm{~ms}$, with a higher standard deviation. $90 \%$ of the samples with the MDVP are below $119.92 \mathrm{~ms}$, whereas using WPCVox they are less than $135.77 \mathrm{~ms}$ (Table 5). The parameter shows a high correlation between both systems (Table 4). Statistically significant differences $(P<0.001)$ have been found between the means using both equipments and separating by gender (Table 3 ).

Regarding the jitt rel, the mean obtained with MDVP is $0.94 \%$, being significantly higher for the females than for the males (Table 5), Results with WPCV ox were significantly different (Table 3), with a mean value of $1.13 \%$. Once again, differences by gender (Table 3) were found (Table 5). The existing correlation between both measurements might be considered high (Table 4).

The mean value with MDVP for RAP is $0.56 \%$ and for $\mathrm{PPQ}$ is $0.55 \%$ (Table 5), below the normative data found in the MDVP manual $\quad(0.68 \%$ for RAP and $0.84 \%$ for PPQ). In the case of WPCVox, the results are slightly higher: 0.69 and $0.66 \%$, respectively (Table 5); being

Table 3 t-test (assuming different variances) to determine whether there is or not difference of means separating by gender

\begin{tabular}{llll}
\hline Parameter & Equipment & Gendet \\
\cline { 3 - 4 } & & MDVP & WPCVox \\
\hline Jitt abs $(\mathrm{ms})$ & Yes* & Yes* & Yes $(P=0.001)$ \\
Jitt rel $(\%)$ & Yes* $^{*}$ & Yes $(P=0.040)$ & Yes* \\
RAP $(\%)$ & Yes* & Yes $(P=0.008)$ & Yes* \\
PPQ $(\%)$ & Yes* & Yes $(P=0.047)$ & Yes* \\
sPPQ $(\%)$ & Yes* & No $(P=0.081)$ & No $(P=0.973)$ \\
Shimm abs (dB) & Yes* & Yes $(P=0.011)$ & Yes $(P=0.004)$ \\
Shimm rel $(\%)$ & Yes* & Yes $(P=0.010)$ & Yes $(P=0.003)$ \\
APQ $(\%)$ & Yes* & Yes* & Yes* \\
sAPQ $(\%)$ & No $(P=0.127)$ & Yes* & Yes* \\
NHR (\%) & Yes* & Yes* & No $(P=0.449)$ \\
VTI $(\%)$ & Yes* & No $(P=0.435)$ & Yes $(P=0.002)$ \\
HNR (dB) & Yes* & Yes* & Yes* \\
NNE $(d B)$ & - & - & Yes* \\
\hline
\end{tabular}

HNR with MDVP is calculated as $10 \log (1 / \mathrm{NHR})$

$* P<0.001$
Table 2 fo (Hz) measured with MDVP and WPCV ox

\begin{tabular}{|c|c|c|c|c|c|c|c|c|c|}
\hline \multirow[t]{2}{*}{ Gender } & \multirow[t]{2}{*}{ Itens } & \multirow{2}{*}{$\begin{array}{l}\mu t \\
\text { MDVP }\end{array}$} & \multirow[t]{2}{*}{$\sigma$} & \multicolumn{2}{|c|}{ Percentile } & \multirow{2}{*}{$\begin{array}{l}\mu \\
\text { WPCVox }\end{array}$} & \multirow[t]{2}{*}{$\sigma$} & \multicolumn{2}{|c|}{ Percentile } \\
\hline & & & & $1 \%$ & $99 \%$ & & & $1 \%$ & $99 \%$ \\
\hline 6 & 222 & 199.98 & 31.4 & 194.50 & 205.47 & 200.35 & 30.9 & 194.97 & 205.75 \\
\hline $8^{x}$ & 126 & 120.56 & 24.8 & 114.77 & 126.35 & 119.70 & 24.6 & 113.97 & 125.43 \\
\hline
\end{tabular}


Table 4 Correlation

measurements among the parameters using MDVP and WPCV ox

$* P<0.001$

\begin{tabular}{lllllll}
\hline Parameter & Kendall & $P$ & Spearman & $P$ & Pearson & $P$ \\
\hline Jitt abs (ms) & 0.807 & $*$ & 0.893 & $*$ & 0.852 & $*$ \\
Jitt rel (\%) & 0.776 & $*$ & 0.873 & $*$ & 0.847 & $*$ \\
RAP $(\%)$ & 0.771 & $*$ & 0.872 & $*$ & 0.847 & $*$ \\
PPQ $(\%)$ & 0.767 & $*$ & 0.865 & $*$ & 0.837 & $*$ \\
sPPQ (\%) & 0.811 & $*$ & 0.889 & $*$ & 0.866 & $*$ \\
Shimm abs (dB) & 0.771 & $*$ & 0.927 & $*$ & 0.928 & $*$ \\
Shimm rel (\%) & 0.769 & $*$ & 0.928 & $*$ & 0.928 & $*$ \\
APQ $(\%)$ & 0.776 & $*$ & 0.933 & $*$ & 0.929 & $*$ \\
sAPQ $(\%)$ & 0.755 & $*$ & 0.910 & $*$ & 0.923 & $*$ \\
NHR $(\%)$ & 0.243 & $*$ & 0.333 & $*$ & 0.308 & $*$ \\
VTI (\%) & 0.119 & 0.003 & 0.161 & 0.003 & 0.156 & 0.004 \\
HNR (dB) & 0.331 & $*$ & 0.470 & $*$ & 0.473 & $*$ \\
\hline
\end{tabular}

closer to the previously mentioned values in the MDVP manual. Smoothing with 55 pitch consecutive periods, a mean value of $\mathrm{SPPQ}=0.77 \%$ is obtained using MDVP, and $0.86 \%$ with WPCVox, both below the normative values found in the MDVP manual (1.02\%). Statistically significant differences have been found between the means of the three parameters using both equipments, and for RAP and PPQ separating by gender (Table 3). Regarding the concordance of the results given by the MDVP and the WPCVox, high correlation coefficients have been found between the mentioned parameters (Table 4). The spreading is higher with WPCVox.

\section{Amplitude perturbation: shimmer paraneters}

The amplitude perturbation measurements, known as shimmer parameters, have a relevant clinical significance as their equivalent in frequency. Despite their importance and clear relationship with the degree of disphony, the study of shimmer has been less extensive in the literature than the study of jitter.

The normative results, separated by gender, are summarized in Table 5. The mean values of shimm abs and shimm rel obtained with MDVP are $0.28 \mathrm{~dB}$ and $3.22 \%$, respectively (Table 5). With WPCVox the results were $0.31 \mathrm{~dB}$ and $3.48 \%$, close to the normative values presented in the MDVP manual $(0.31 \mathrm{~dB}$ and $3.81 \%)$. The smoothing with 11 and 55 pitch periods gives mean values of $\mathrm{APQ}=2.39 \%$ and $\mathrm{SAPQ}=4.61 \%$ using MDVP, and $\mathrm{APQ}=2.57 \%$ and $\mathrm{SAPQ}=4.72 \%$ with WPCVox. The shimmer parameters revealed high correlation coefficients (Table 4). There are statistically significant differences separating by gender for both equipments and there are also differences between the two programs, except for sAPQ (Table 3). The spreading of the values is higher with WPCVox.

\section{Noise measurements}

The noise parameters measure the degree of hoarseness caused by air turbulences introduced by the presence of a disorder. There are many noise parameters reported in the literature and WPCV ox and MDVP provide different noise measurements (see Table 1). Those measurements that seem to be equivalent in both systems -NHR and VTI- have been directly compared. Also, the HNR calculated with WPCVox has been transformed using $10 \log (1 / \mathrm{HNR})$ to be compared with the NHR given by MDVP. This transformation has been carried out previously by other authors

The normative results, separated by gender, are summarized in Table 5. The normative values obtained for the NHR using both equipments differ enormously (Table 3): MDVP gave mean values around $0.13 \%$ (above the normative data reported in the MDVP manual), whereas WPCVox gave $1.05 \%$ on average (Table 5). In contrast with WPCVox, there have been found statistically significant differences of means separating the dataset by gender using MDVP (Table 3). Another difference found is related with the shape of the distribution: the values obtained with MDVP follow a Gaussian distribution, whereas the data obtained with WPCVox do not. Focusing on the correlation between the data obtained with WPCV ox and MDVP, there is a statistically significant weak relationship between both measurements (Table 4).

Regarding the VTI, the normative values obtained using both equipments also differ greatly (Table 3): MDVP gave mean values around $0.04 \%$, whereas WPCV ox gave $0.51 \%$ on average (Table 5). In contrast with NHR, statistically significant differences of means have been found with WPCVox separating the dataset by gender, but not with MDVP (Table 3). The correlation between both series of VTI demonstrates that there exists a very weak relationship between the measurements (Table 4 ). 
Table 5 Normative values of the parameters calculated with MDVP and WPCVox

\begin{tabular}{|c|c|c|c|c|c|c|}
\hline \multirow[t]{2}{*}{ Normal voices } & \multicolumn{2}{|l|}{$90^{n}$} & \multicolumn{2}{|l|}{6} & \multicolumn{2}{|l|}{$5^{n}$} \\
\hline & MDVP & WPCVox & MDVP & WPCVox & MDVP & WPCVox \\
\hline \multicolumn{7}{|l|}{ Jitt abs (ms) } \\
\hline$\mu$ & 58.85 & 67.77 & 51.30 & 62.55 & 72.25 & 77.08 \\
\hline$\sigma$ & 42.61 & 50.47 & 37.27 & 47.84 & 48.03 & 53.80 \\
\hline Mode & 23.91 & 24.44 & 23.91 & 24.44 & 39.56 & 15.63 \\
\hline Percentile $75 \%$ & 78.82 & 89.24 & 68.52 & 86.29 & 93.51 & 98.53 \\
\hline Percentile $90 \%$ & 119.92 & 135.77 & 109.82 & 126.80 & 154.55 & 169.54 \\
\hline \multicolumn{7}{|l|}{ Jitt tel $(\%)$} \\
\hline$\mu$ & 0.94 & 1.13 & 0.99 & 1.24 & 0.84 & 0.96 \\
\hline$\sigma$ & 0.65 & 0.89 & 0.70 & 0.94 & 0.54 & 0.78 \\
\hline Mode & 0.38 & 0.45 & 1.36 & 0.45 & 0.31 & 0.48 \\
\hline Petcentile $75 \%$ & 1.22 & 1.50 & 1.39 & 1.65 & 1.02 & 1.08 \\
\hline Petcentile $90 \%$ & 1.89 & 2.27 & 2.03 & 2.36 & 1.71 & 2.05 \\
\hline \multicolumn{7}{|l|}{$\operatorname{RAP}(\%)$} \\
\hline$\mu$ & 0.56 & 0.69 & 0.60 & 0.76 & 0.49 & 0.57 \\
\hline$\sigma$ & 0.40 & 0.55 & 0.43 & 0.57 & 0.33 & 0.48 \\
\hline Mode & 0.25 & 0.27 & 0.25 & 0.29 & 0.25 & 0.18 \\
\hline Petcentile $75 \%$ & 0.73 & 0.93 & 0.85 & 1.01 & 0.62 & 0.67 \\
\hline Percentile $90 \%$ & 1.15 & 1.41 & 1.21 & 1.47 & 1.01 & 1.23 \\
\hline \multicolumn{7}{|l|}{$\mathrm{PPQ}(\%)$} \\
\hline$\mu$ & 0.55 & 0.66 & 0.58 & 0.72 & 0.49 & 0.56 \\
\hline$\sigma$ & 0.38 & 0.51 & 0.41 & 0.54 & 0.32 & 0.44 \\
\hline Mode & 0.21 & 0.31 & 0.24 & 0.23 & 0.26 & 0.26 \\
\hline Percentile $75 \%$ & 0.71 & 0.89 & 0.78 & 0.98 & 0.58 & 0.63 \\
\hline Petcentile $90 \%$ & 1.12 & 1.29 & 1.16 & 1.36 & 1.02 & 1.20 \\
\hline \multicolumn{7}{|l|}{$\mathrm{sPPQ}(\%)$} \\
\hline$\mu$ & 0.77 & 0.86 & 0.75 & 0.87 & 0.80 & 0.86 \\
\hline$\sigma$ & 0.41 & 0.50 & 0.40 & 0.51 & 0.41 & 0.48 \\
\hline Mode & 0.48 & 0.36 & 0.48 & 0.37 & 0.88 & 0.36 \\
\hline Petcentile $75 \%$ & 0.95 & 1.04 & 0.96 & 1.06 & 0.91 & 1.01 \\
\hline Percentile $90 \%$ & 1.28 & 1.49 & 1.31 & 1.48 & 1.26 & 1.56 \\
\hline \multicolumn{7}{|l|}{ Shimm abs (dB) } \\
\hline$\mu$ & 0.28 & 0.31 & 0.27 & 0.29 & 0.31 & 0.39 \\
\hline$\sigma$ & 0.14 & 0.14 & 0.12 & 0.12 & 0.16 & 0.17 \\
\hline Mode & 0.28 & 0.23 & 0.28 & 0.21 & 0.23 & 0.23 \\
\hline Percentile $75 \%$ & 0.33 & 0.37 & 0.32 & 0.36 & 0.38 & 0.41 \\
\hline Percentile $90 \%$ & 0.47 & 0.51 & 0.40 & 0.46 & 0.52 & 0.58 \\
\hline \multicolumn{7}{|l|}{ Shimm rel (\%) } \\
\hline$\mu$ & 3.22 & 3.48 & 3.06 & 3.27 & 3.53 & 3.86 \\
\hline$\sigma$ & 1.57 & 1.61 & 1.41 & 1.40 & 1.78 & 1.87 \\
\hline Mode & 1.69 & 2.25 & 1.69 & 2.25 & 2.85 & 3.05 \\
\hline Percentile $75 \%$ & 3.81 & 4.22 & 3.70 & 4.07 & 4.31 & 4.77 \\
\hline Percentile $90 \%$ & 5.45 & 5.81 & 4.64 & 5.22 & 5.99 & 6.56 \\
\hline \multicolumn{7}{|l|}{$\mathrm{APQ}(\%)$} \\
\hline$\mu$ & 2.39 & 2.57 & 2.19 & 2.35 & 2.75 & 2.98 \\
\hline$\sigma$ & 1.08 & 0.15 & 0.96 & 1.00 & 1.19 & 1.28 \\
\hline Mode & 1.48 & 1.13 & 1.48 & 1.31 & 3.13 & 1.54 \\
\hline Petcentile $75 \%$ & 282 & 3.08 & 2.56 & 2.81 & 3.20 & 3.64 \\
\hline
\end{tabular}


Table 5 continued

\begin{tabular}{|c|c|c|c|c|c|c|}
\hline \multirow[t]{2}{*}{ Normal voices } & \multicolumn{2}{|l|}{03} & \multicolumn{2}{|l|}{9} & \multicolumn{2}{|l|}{3} \\
\hline & MDVP & WPCVox & MDVP & WPCVox & MDVP & WPCVox \\
\hline Petcentile $90 \%$ & 3.94 & 4.16 & 3.19 & 3.68 & 4.39 & 4.80 \\
\hline \multicolumn{7}{|l|}{$\mathrm{SAPQ}(\%)$} \\
\hline$\mu$ & 4.61 & 4.72 & 4.32 & 4.37 & 5.12 & 5.34 \\
\hline$\sigma$ & 1.90 & 2.03 & 1.67 & 1.67 & 2.17 & 2.44 \\
\hline Mode & 2.53 & 3.10 & 2.76 & 4.14 & 3.61 & 3.71 \\
\hline Percentile $75 \%$ & 5.63 & 5.67 & 5.36 & 5.26 & 6.70 & 6.54 \\
\hline Percentile $90 \%$ & 7.23 & 7.50 & 6.72 & 6.74 & 7.81 & 8.62 \\
\hline \multicolumn{7}{|l|}{ NHR $(\%)$} \\
\hline$\mu$ & 0.13 & 1.05 & 0.12 & 1.00 & 0.13 & 1.14 \\
\hline$\sigma$ & 0.02 & 1.92 & 0.02 & 1.91 & 0.02 & 1.93 \\
\hline Mode & 0.13 & 0.03 & 0.12 & 0.03 & 0.13 & 0.13 \\
\hline Percentile $75 \%$ & 0.14 & 1.10 & 0.13 & 1.07 & 0.15 & 1.28 \\
\hline Percentile $90 \%$ & 0.15 & 2.89 & 0.14 & 2.62 & 0.16 & 3.27 \\
\hline \multicolumn{7}{|l|}{ VTI $(\%)$} \\
\hline$\mu$ & 0.04 & 0.51 & 0.04 & 0.40 & 0.04 & 0.70 \\
\hline$\sigma$ & 0.01 & 0.92 & 0.01 & 0.76 & 0.02 & 1.13 \\
\hline Mode & 0.04 & 0.03 & 0.04 & 0.02 & 0.04 & 0.07 \\
\hline Percentile $75 \%$ & 0.05 & 0.56 & 0.05 & 0.42 & 0.05 & 0.75 \\
\hline Petcentile $90 \%$ & 0.06 & 1.40 & 0.06 & 1.11 & 0.06 & 1.93 \\
\hline \multicolumn{7}{|l|}{ HNR (dB) } \\
\hline$\mu$ & 29.07 & 25.62 & 29.23 & 26.29 & 28.79 & 24.40 \\
\hline$\sigma$ & 0.77 & 3.53 & 0.75 & 3.35 & 0.75 & 3.53 \\
\hline Mode & 29.36 & 26.01 & 29.36 & 26.01 & 28.79 & 21.95 \\
\hline Petcentile $75 \%$ & 29.51 & 28.07 & 29.59 & 28.66 & 29.16 & 26.59 \\
\hline Percentile $90 \%$ & 30.19 & 30.23 & 30.31 & 30.68 & 29.78 & 29.58 \\
\hline \multicolumn{7}{|l|}{ NNE (dB) } \\
\hline$\mu$ & - & -15.28 & - & -16.44 & - & -13.21 \\
\hline$\sigma$ & - & 0.92 & - & 4.73 & - & 18.17 \\
\hline Mode & - & -21.48 & - & -26.87 & - & -9.08 \\
\hline Percentile $75 \%$ & - & -11.97 & - & -12.89 & - & -9.96 \\
\hline Percentile $90 \%$ & - & -8.69 & - & -9.78 & - & -7.89 \\
\hline
\end{tabular}

With respect to HNR, the normative values obtained using both equipments also differ from the statistical point of view (Table 3): MDVP gave mean values around $29.07 \mathrm{~dB}$, whereas WPCVox gave $25.62 \mathrm{~dB}$ on average (Table 5). Moreover, statistically significant differences of means have been found both with WPCVox and MDVP separating the dataset by gender. The study of the correlation between both series of HNR demonstrates that there exists only a weak relationship between both measurements (Table 4).

Regarding the measurements of NNE made with WPCVox, no comparisons can be carried out because MDVP do not provide such a parameter. Its normative values are summarized in (Table 5). Once again, the spreading of the values is clearly higher with WPCVOx.
Discriminative capabilities

The discriminative capability of the acoustic parameters given by WPCVox and MDVP has been tested and compared. This test has been carried out with the Voice Disorders Database distributed by Kay Elemetrics. The parameters listed in Table 6 were computed for every register, and the results analysed in two ways: comparing the probability distributions of normal and pathological voices for each parameter and computing the receiver operating characteristics (ROC) curves (Fig. 1) . The ROC is analyzed calculating the area under the curve (AUC) and the standard error (SE) of the area as suggested

Table 6 shows the classification rate of the parameters obtained from the histograms at the threshold value that 
Table 6 Classification rates, AUC and SE for the set of parameters calculated with MDVP and WPCVox

\begin{tabular}{|c|c|c|c|c|c|c|c|}
\hline \multirow[t]{2}{*}{ Type } & \multirow[t]{2}{*}{ Parameter } & \multicolumn{2}{|c|}{ Classification tate $(\%)$} & \multicolumn{2}{|l|}{ AUC } & \multicolumn{2}{|l|}{ SE } \\
\hline & & MDVP & WPCV ox & MDVP & WPCVox & MDVP & WPCVox \\
\hline \multirow{5}{*}{$\begin{array}{l}\text { Frequency } \\
\text { perturbation }\end{array}$} & Jitt abs (ms) & 83.41 & 82.24 & 0.89 & 0.86 & 0.021 & 0.025 \\
\hline & Jitt tel $(\%)$ & 75.11 & 73.83 & 0.84 & 0.80 & 0.027 & 0.031 \\
\hline & $\operatorname{RAP}(\%)$ & 75.11 & 74.30 & 0.84 & 0.79 & 0.027 & 0.032 \\
\hline & $\mathrm{PPQ}(\%)$ & 74.19 & 73.40 & 0.84 & 0.79 & 0.026 & 0.032 \\
\hline & $\mathrm{sPPQ}(\%)$ & 76.49 & 75.70 & 0.84 & 0.82 & 0.027 & 0.030 \\
\hline \multirow{4}{*}{$\begin{array}{l}\text { Amplitude } \\
\text { perturbation }\end{array}$} & Shinm abs (dB) & 83.41 & 82.71 & 0.92 & 0.92 & 0.018 & 0.019 \\
\hline & Shimm rel $(\%)$ & 83.41 & 83.64 & 0.92 & 0.92 & 0.018 & 0.019 \\
\hline & $\mathrm{APQ}(\%)$ & 84.79 & 85.04 & 0.92 & 0.92 & 0.018 & 0.020 \\
\hline & SAPQ $(\%)$ & 79.26 & 80.84 & 0.90 & 0.90 & 0.021 & 0.021 \\
\hline \multirow[t]{4}{*}{ Noise } & $\operatorname{NHR}(\%)$ & 79.26 & 78.90 & 0.88 & 0.84 & 0.022 & 0.027 \\
\hline & VTI $(\%)$ & 63.13 & 75.70 & 0.72 & 0.84 & 0.037 & 0.027 \\
\hline & $\operatorname{HNR}(\mathrm{dB})$ & 79.26 & 84.57 & 0.88 & 0.92 & 0.022 & 0.027 \\
\hline & NNE (dB) & - & 89.71 & - & 0.94 & - & 0.014 \\
\hline
\end{tabular}

separates optimally normal and pathological voices. Moreover, the AUC curve and the SE are presented. In general, the SE shows that the AUC presents a clear overlapping for both systems, so we cannot conclude that there exist evidences that neither WPCVox nor MDVP have better discrimination capabilities. Figure 1 shows the ROC curves for all the parameters extracted with MDVP and WPCVox.

\section{Discussion}

The discussion is organized into two sections following the same scheme used to present the results. The first is a discussion of the similarities between the parameters obtained with both systems and the normative values. The second discusses the discrimination capabilities of both equipments.

\section{Normative values}

\section{Fundamental frequency}

As expected, the variability is higher in the male group than for the females. This fact is clearly reflected in the range and standard deviation calculated (Table 2). The high correlation between the values obtained with both equipments must be emphasized. The mean values and the standard deviation found (Table 2) are very similar to those reported in the state of the art Moreover, the results are in concordance with those obtained by Karnell

\section{Frequency perturbation parameters}

Among the frequency perturbation parameters, the jitt abs, measured in ms (absolute value), has been one of the parameters most widely used by clinicians, with a long tra- dition in scientific literature . The results obtained for MDVP and WPCVox regarding the $90 \%$ percentile are slightly above the normative values given in the MDVP manual $(83.2 \mathrm{~ms})$ and match well with the results published by other authors who used Spanish speakers

The values of jitt rel obtained are higher than those obtained by other researchers: Hollien obtained $0.48 \%$ for males with a fo around $102 \mathrm{~Hz}$, and $0.76 \%$ for males with fo around $142 \mathrm{~Hz}$. Orlikoff obtained $0.42 \%$ for males aged between 26 and 33 years, and González obtained $0.94 \%$ for the remales and $0.68 \%$ for the males subset, using young Spanish speakers with a significant sample size ( $n=148$ ). Also, the normative values obtained for the jitt rel are higher than those found in other studies done in Spain . However, these studies were carried out with another analysis system (Dr, Speech, by Tiger Electronics).

Fernández , used a dataset of 154 non-smoking adults, finding a value of $P P Q=0.23$; the group of smokers in Damborenea yielded $P P Q=0.27$. A wide sample of former studies revealed RAP values lower than those obtained in this work. As examples, Preciado's study , using a control group of 64 Spanish teachers, obtained RAP $=0.35$; the work of Walton and Orlikoff , obtained RAP $=0.28$; Dwire and McCauley, with North American patients, obtained RAP $=0.38$ for males and $\mathrm{RAP}=0.89$ for females, both groups in the age range of 18-25; Takahashi and Koike , using Japanese speakers, achieved a RAP $=0.57$ for males and $\mathrm{RAP}=0.61$ for females.

The significant differences found between genders in the frequency perturbation parameters confirm what is

\footnotetext{
${ }^{3}$ The notmative values in the MDVP manual were obtained with English speakers from the sustained phonation of vowel 'ah'
} 

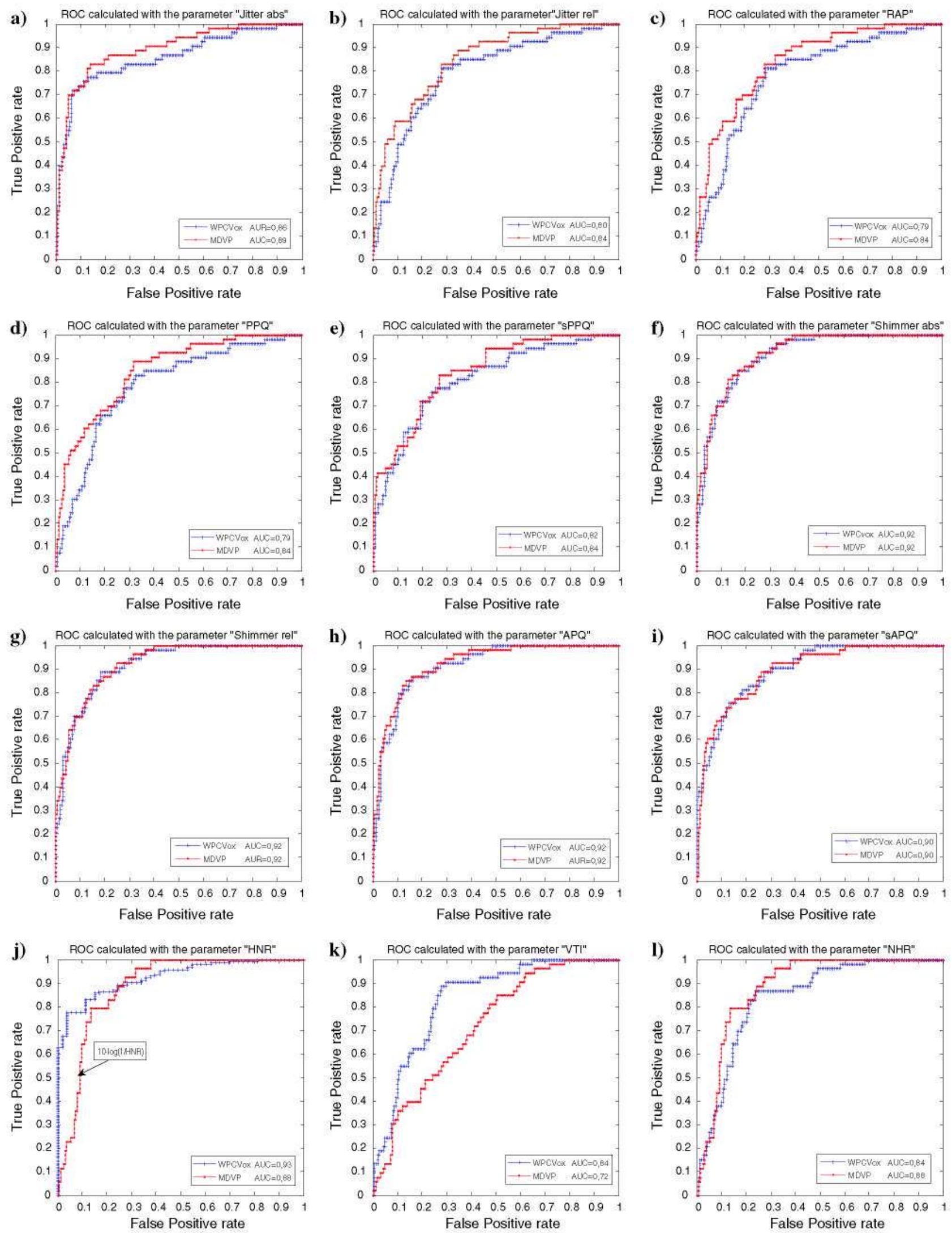

Fig. 1 Receiver operating characteristic curves for the parameters extracted with MDVP and WPCV ox; a jitt abs; b jitt rel; $\mathbf{c}$ RAP; $\mathbf{d}$ PPQ; e sPPQ; f shimm abs; g shimm rel; h APQ; i sAPQ; $\mathbf{j}$ HNR; $\mathbf{k}$ VTI; 1 NHR 
expressed regarding these differences: "the preliminary evidence points out to the possibility that the adult females could have more vocal jitter than males, at least for several vowels. Fortunately, the observed differences between genders are not big enough to provide a diagnostic error". This tact is also affected by the difference that exists between males and females regarding the fo; despite the normalization carried out according to the fo value, there still exist a minor influence.

The tact that WPCVox has just being available in the market makes it difficult to find previous works using this tool in existing literature. There do, however, exist works carried out with other tools: Karnell compared three analysis systems, finding discrepancies in the results obtained for the jitter and shimmer parameters (with a low correlation coefficient $r=0.45$ ). The only parameter that revealed great correlation was the fo. These results revealed that the equipments compared vary more between themselves than do the MDVP and WPCVox. The same study revealed that the correlation between the perturbation parameters obtained were partially improved by rejecting the sample outlayers. In any case, the correlation index was lower than the correlation given between MDVP and WPCVox.

Despite of the differences of the mean values, the high correlation coefficients obtained by comparing the frequency perturbation results between MDVP and WPCVox, and the fact that the parameters are calculated using the same algorithms in both systems, revealed a great concordance. The differences have been laid down to the fact that the algorithms are based on the same grounds but small differences must be expected in their implementation.

\section{Amplitude perturbation parameters}

Horii used for his study the 'a' vowel uttered by 31 males and 20 females, obtaining shimm abs equal to $0.47 \mathrm{~dB}$ and $0.33 \mathrm{~dB}$, respectively, with no significant differences among genders. In our study, $0.31 \mathrm{~dB}$ were obtained for the males group with WPCVox and $0.39 \mathrm{~dB}$ for the females, with statistically significant differences between the genders. The sample size must be responsible for the differences. Orlikoff and Kahane reported an average for normal male voices around $0.31 \mathrm{~dB}$.

Comparing with other studies that used a corpus of Spanish speakers, in González, the $90 \%$ of the shimm abs values were below $0.53 \mathrm{~dB}$ and shimm rel values below $5.98 \%$. These ranges are smaller when analyzing our dataset with both programs (Table 5).

The non smoking patients in Fernández achieved an average $\mathrm{APQ}=2.02 \%$; the smoking patients in Damborenea $\quad 2.56 \%$; González reported $A P Q=2.95 \%$ and $s \mathrm{APQ}=5.02 \%$. These values are close to the values obtained in this study and congruent with the normative data described in the literature.

The high correlation coefficients obtained by comparing the amplitude perturbation results among MDVP and WPCVox, and the fact that the parameters are calculated based on the same algorithms in both systems, revealed a great concordance. Once again, the differences have been laid down to the fact that the algorithms are based on the same grounds but small differences must be expected.

\section{Noise measurements}

It has been amply demonstrated that the parameters based on harmonic energy and noise have an extensive relationship with the physiopathological mechanism of disphony.

The average NHR measured with the MDVP is $0.13 \%$ and with WPCV ox $1.05 \%$. A total of $75 \%$ of the population studied with MDVP reported values below 0.14 and $90 \%$ below $0.15 \%$. These values are below the normative data given by the MDVP manual. The differences of the NHR between females $(0.12 \%)$ and males $(0.13 \%)$ using MDVP is statistically significant. The González study reported very similar results and statistical significances. Focusing on the correlation between the data obtained with WPCV and MDVP, statistically significant similarities have been found to ensure that there is a weak relationship between both measurements.

The VTI measures noise turbulences caused by an incomplete closure of the vocal folds. Hence, most of these noise components are in the high frequency band. The results revealed in our study gave a mean value of $0.04 \%$ using MDVP, very similar to the results found in González $: 0.05 \%$. Also, González concluded the same about the differences between gender with MDVP. The $75 \%$ of the subjects revealed a score below $0.05 \%$; and $90 \%$ below $0.06 \%$. Similar results were reported in the MDVP manual. The spreading of the values obtained with WPCVox is 7 times more than the spreading found with MDVP, and the mean values are higher. There is a clear variation between their mean, standard deviation and the shape of the distribution. Moreover, the study of the correlation between both series of VTI demonstrates that there exists a very weak relationship between both measurements.

The comparison between the HNR given by WPCV ox and the transformed HNR of MDVP also demonstrated a weak relationship.

The explanation to the weak relationship is that the noise parameters given by both equipments are based on similar principles but measure different aspects of the phenomenon. In contrast to the amplitude and frequency perturbation features, the differences found in the noise parameters stands on the algorithms used by WPCVox and MDVP: whereas MDVP is using the algorithms presented , WPCVox 
Fig. 2 Bar plot showing the classification tate of each acoustic parameter measured with MDVP and WPCVox

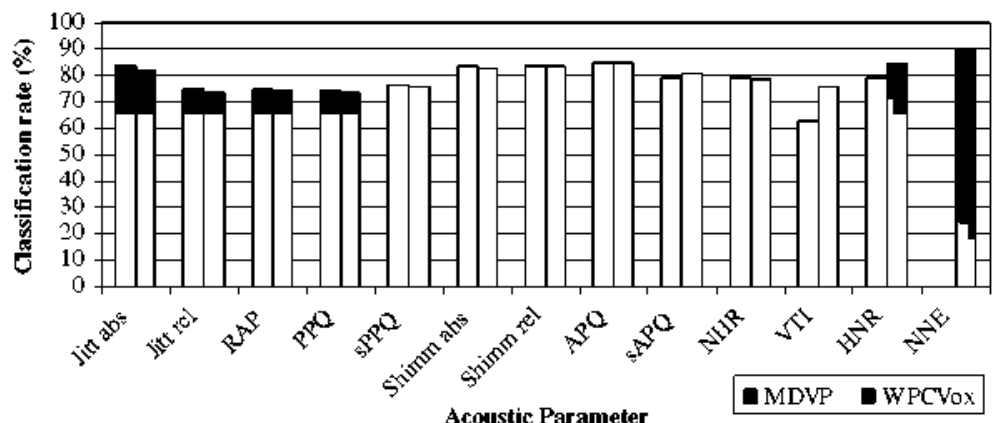

calculates the noise parameters following the scheme presented . The efficiency of these algorithms has been tested before by their authors, so it will not be treated in this paper. Despite of the differences found, the validity and discrimination capabilities of these algorithms for the clinical diagnosis is clear and will be commented in the next section.

\section{Discrimination capabilities}

The results demonstrate the ability to discriminate between normal and pathological voices using the parameters calculated with WPCVox and MDVP. In general, the ability to discriminate between normal and pathological voices may be considered very similar using both equipments. MDVP provides slightly better discrimination capacities using the frequency perturbation parameters, whereas WPCVox seems to work better with noise parameters. The amplitude perturbation parameters showed almost the same discrimination abilities with both equipments.

In spite of the above mentioned differences in measuring the HNR, both equipments showed a clear discrimination capability for this parameter. Also, despite the differences in measuring the VTI, both equipments showed clear discrimination capabilities, with WPCVox being considerably superior for this parameter. Regarding the HNR of WPCVox, it demonstrated better discrimination ability than using the transformed HNR given by MDPV.

The discrimination ability of the parameter NNE is remarkable $(89.71 \%)$. Such a parameter is not calculated by MDVP.

Figure 2 summarises the discrimination capabilities of the parameters obtained with both systems.

\section{Conclusions}

The aim of the study is to prove neither the efficiency nor the accuracy of the algorithms for the detection of voice disorders because they were tested before by other authors. The objective is just to compare the measurements of MDVP and WPCVOx to find out whether the values obtained with both equipments are comparable or not, and to know how large are the differences due to the implementation of the algorithms.

In general terms, the results concluded that WPCVox provides measurements very similar to those obtained with MDVP. The differences found do not demonstrate that WPCVox performs worse or better than MDVP. Moreover, the ROCs demonstrate that the discrimination abilities of both systems are very similar.

Regarding the amplitude and frequency perturbation parameters, the results obtained are also comparable to those found in the literature based on Spanish speakers with other equipments. When we observe the correlation index and compare the acoustic parameters calculated with MDVP and WPCVox, and keeping in mind that both systems are developed following the same algorithms, we can state that there are clear similarities measuring the fo and the amplitude and frequency perturbation parameters using both systems.

The worst results in terms of correlation were obtained for the noise parameters: NHR, VTI and HNR. No statistical evidence has been found to indicate that both systems measure the same aspects of the phenomena with these parameters. These differences are explained because the noise parameters of both systems are based on different algorithms. Despite of the differences, it has been demonstrated that the noise measurements of WPCVox have a clear discrimination potential, even better than those of MDVP.

The parameters obtained with WPCVox are reliable for the discrimination between normal and pathological voices, with classification rates around $80 \%$ for each parameter alone. WPCVox gives two acoustic parameters (NNE and HNR) not present in MDVP. As reported in the literature, these parameters have revealed great discrimination abilities (greater than the amplitude and frequency perturbation parameters). The NNE's classification rate over $89 \%$ must be highlighted.

In conclusion, the matching of most parameters extracted with both equipments (except NHR, VTI and HNR) allows the data transter possible among them, so those patients evaluated with MDVP could be evaluated 
and compared accurately with WPCVox (and vice versa) obtaining very similar results.

Acknowledgments This research was partially catried out under grants: TIC2003-08956-C02-00 from the Ministry of Education of Spain, and AL06-EX-PID-033 fron the Universidad Politécnica de Madrid, Spain

\section{References}

Kay Elemetrics Corporation (1994) MDVP operations manual. Model 4305

Godino-Llorente JI, Sáenz-Lechón N, Osma-Ruiz V et al (2006) An integrated tool for the diagnosis of voice disorders. Med Eng Phys 28:276-289

Smits I, Ceuppens P. de Bodt M (2005) A comparative study of acoustic voice measurements by means of Dr. Speech and Computerized Speech Lab. J Voice 19:187-196

Hirano M (1981) Psycho-acoustic evaluation of voice. Springer, New York

Kay Elemetrics Corp (1994) Voice Disorders Database, version 1.03. Lincoln Park, NJ

Parsa V. Jamieson D (2000) Identification of pathological voices using glottal noise measures. J Speech Lang Hear Res 43:469-485 Childers D (2000) Speech processing and synthesis toolboxes. Wiley, New York

Baken RJ, Orlikoff R (2000) Clinical measurement of speech and voice. 2nd edn. Singular Publishing Group. San Diego. CA. USA

Feijoo S, Hernández-Espinosa C (1990) Short-tern stability measures for the evaluation of vocal quality. J Speech Hear Res $33: 324-334$

Deliyski D (1993) Acoustic model and evaluation of pathological voice production. In: Proceedings of Eurospeech'93. Betlin. Germany $3: 1969-1972$

Yumoto E, Gould W, Baer T (1982) Harmonics-to-noise ratio as an index of the degree of hoarseness. J Acoust Soc Am 71:15441550

de Krom G (1993) A cepstrum-based technique for determining a hatmonics-to-noise ratio in speech signals. J Speech Heat Res $36: 254-266$

Kasuya H, Ogawa S, Mashima K, Ebihara S (1986) Normalized noise energy as an acoustic measure to evaluate pathologic voice. J Acoust Soc Am 80:1329-1334

Weiss NA (2000) Introductory statistics. 6th edn. Addison Wesley. Reading, USA

Michaelis D. Fröhlich M. Strube HW (1998) Selection and combination of acoustic features for the description of pathologic voices. J Acoust Soc Am 103:1628-1639
Fröhlich M. Michaelis D. Strube HW, Kruse E (2000) Acoustic voice analysis by means of the hoarseness diagram. J Speech Lang Hear Res 43:706-720

Hanley J, McNeil B (1983) A method of comparing the areas under receiver operating characteristics curves derived from the same cases. Radiology 148:839-843

Fernandez R, Dambotenea J et al (1999) Acoustic analysis of the normal voice in nonsmoking adults. Acta Otorrinolatingol Española $50: 134-141$

Damborenea J. Fernández R et al (1999) The effect of tobacco consumption on acoustic voice analysis. Acta Otorrinolaringol Española $50: 448-452$

Karnell MP, Hall KD. et al (1995) Comparison of fundamental frequency and perturbation measurements among three analysis systens. J Voice 9:383-393

Baker K. Ramig L. Jones S. Freed CR (1997) Preliminary voice and speech analysis following fetal dopamine transplants in 5 individuals with Parkinson disease. J Speech Lang Hear Res 40:615-626

McAllister A, Sundberg J, Hibi S (1998) Acoustic measurements and perceptual evaluation of hoarseness in children's voices. Logopedics Phoniatrics Vocol 23:27-38

Wolfe V, Cornell R, Palmer C (1991) Acoustic correlates of pathologic voice types. J Speech Hear Res 34(3):509-516

Wolfe V, Martin D (1997) Acoustic cottelates of dysphonia: type and severity. J Commun Disotd 30(5):403-416

Preciado JA. Fernandez S (1998) Digital analysis of the acoustic signal in vocal pathology diagnosis. Sensitivity and specificity of shimmer and jitter measurements. Acta Otorrinolaringol Española 49:475-481

Hollien H, Thompson CL, Cannon B (1973) Speech intelligibility as a function of ambient pressute and Heo 2 atmosphere. Aerosp Med 44:249-253

Orlikotf R, Baken RJ (1990) Considerations on the relationship between the fundamental frequency of phonation and vocal jitter. Folia Phoniatrica 42:31-40

González T. Cervera T. Miralles J (2002) Análisis acústico de la voz: fiabilidad de un conjunto de parámetros multidimensionales. Acta Otortinolaringol Española 53:256-268

Orlikotf R, Walton JH (1994) Speaker race identification from acoustic cues in the vocal signal. J Speech Hear Res 37:738-745 Dwire A. McCauley R (1995) Repeated measures of vocal fundamental frequency perturbation obtained using the Visi-pitch. J Voice $9(2) \div 156-162$

Takabashi H. Koike Y (1976) Some perceptual dimensions and acoustical correlates of pathologic voices. Acta Otolaryngol 338:1-24

Karnell MP (1991) Latyngeal perturbation analysis: mininum length of analysis window. J Speech Hear Res 34:544-548

Horii Y (1979) Fundamental frequency perturbation observed in sustained phonation. J Speech Heat Res 22:5-19

Orlikoff R, Kahane JC (1991) Influence of mean sound pressure level on jitter and shimmer measures. J Voice 5:113-119 